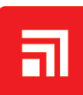 CHITKARA \\ Journal of Nuclear Physics, Material Sciences, Radiation and Applications \\ Journal homepage: https://jnp.chitkara.edu.in/
}

\section{Response to Neutrons and $\gamma$-rays of Two Liquid Scintillators}

\author{
Hector Rene Vega-Carrillo ${ }^{1 *}$, Martha Isabel Escalona-Llaguno ${ }^{1}$, Luis Hernandez-Adame ${ }^{2}$, Sergio M. Sarmiento-Rosales ${ }^{1}$, Claudia A. Márquez- \\ Mata $^{1}$, Guillermo E. Campillo-Rivera ${ }^{1}$, V.P. Singh ${ }^{3}$, Teodoro Rivera-Montalvo ${ }^{4}$ and Segundo Agustin Martínez-Ovalle ${ }^{5}$ \\ ${ }^{1}$ Academic Unit of Nuclear Studies of the University Autonomous of Zacatecas, C. Cipres 10, Fracc. La Peñuela, 98060 Zacatecas, \\ Zac. Mexico \\ ${ }^{2}$ CONACyT - Center for Biological Research of the Northwest, S.C., Av. Instituto Politecnico Nacional 195, Col. Playa Palo de Santa \\ Rita Sur 23090 La Paz, BCS. Mexico \\ ${ }^{3}$ Karanatak University, Dharwad, Karnataka, India-580003 \\ ${ }^{4}$ Center for Research in Applied Science and Advanced Technology - Legaria Unit of IPN, Av. Legaria 694, Col. Irrigación, 11500 \\ Ciudad de Mexico, Mexico \\ ${ }^{5}$ Pedagogical and Technological University of Colombia, Tunja-150003, Colombia
}

*Email: fermineutron@yahoo.com

\section{ARTICLE INFORMATION}

Received: August 20, 2018

Revised: January 5, 2019

Accepted: February 8, 2019

Published online: February 26, 2019

\section{Keywords:}

Response, Liquid scintillator, Detectors,

Neutrons, Gamma-rays, UltimaGold,

Optiphase

DOI: $10.15415 /$ jnp.2019.62025

\begin{abstract}
UltimaGold ${ }^{\mathrm{TM}} \mathrm{AB}$ and OptiphaseTrisafe are two liquid scintillators made by Perkin Elmer and EG \& G Company respectively. Both are commercially promoted as scintillation detectors for $\alpha$ and $\beta$ particles. In this work, the responses to $\gamma$-rays and neutrons of UltimaGold ${ }^{\mathrm{TM}} \mathrm{AB}$ and OptiphaseTriSafe liquid scintillators, without and with reflector, have been measured aiming to use these scintillators as $\gamma$-rays and neutron detectors. Responses to $\gamma$-rays and neutrons were measured as pulse shape spectra in a multichannel analyzer. Scintillators were exposed to gamma rays produced by ${ }^{137} \mathrm{Cs},{ }^{54} \mathrm{Mn},{ }^{22} \mathrm{Na}$ and ${ }^{60} \mathrm{Co}$ sources. The response to neutrons was obtained with a ${ }^{241} \mathrm{AmBe}$ neutron source that was measured to 25 and $50 \mathrm{~cm}$ from the scintillators. The pulse height spectra due to gamma rays are shifted to larger channels as the photon energy increases and these responses are different from the response due to neutrons. Thus, UltimaGold ${ }^{\mathrm{TM}} \mathrm{AB}$ and OptiphaseTrisafe can be used to detect $\gamma$-rays and neutrons.
\end{abstract}

\section{Introduction}

Outside the atomic nucleus neutrons are unstable. Neutrons are produced artificially in anthropogenic activities, as well as naturally, mainly during the interaction of cosmic rays with nuclei in the atmosphere; also, neutrons are produced during thunderstorm storm, as well as in nuclear reactions between alpha particles and the nuclei of the earth $[1,2]$.

In some interactions with matter neutrons generate secondary charged particles and photons. Due to its radiobiological efficiency and the way in which it interacts with matter, it is important to determine, by means of calculations and/or measurements, the neutron energy distribution or neutron spectrum [3]. In order to measure the neutron spectrum it is necessary to use neutron detectors such as activation foils, proportional counter $\left({ }^{10} \mathrm{BF}_{3}\right.$ and $\left.{ }^{3} \mathrm{He}\right)$, bubble detectors, inorganic, and organic scintillators $[4,5]$.

Organic scintillators (liquid or plastic) have fluorescent materials dissolved in a solvent base. Fluorescent materials are aromatic hydrocarbons whose mean atomic number varies from 3.5 to 5 . The most commonly used fluorescent materials are the PTP $\left(\mathrm{C}_{18} \mathrm{H}_{14}\right)$, B-PBD $\left(\mathrm{C}_{24} \mathrm{H}_{22} \mathrm{~N}_{2 \mathrm{O}}\right)$, PPO $\left(\mathrm{C}_{15} \mathrm{H}_{11} \mathrm{NO}\right)$, and POPOP $\left(\mathrm{C}_{24} \mathrm{H}_{16} \mathrm{~N}_{2} \mathrm{O}_{2}\right)$. In the interaction of ionizing radiation with the fluorescent organic materials released energy excites the aromatic solvent molecules. Neutrons are used in basic science (which involves raising and answering research questions), also are used in fusion, fission, to detect special nuclear materials, in dosimetry and characterization of materials [5].

Knowing the fluence rate (flux) and energy distribution of neutrons is important in its use as a neutron source in molten salt nuclear reactors [6]. Also, during calibration and neutron analysis of deuterium plasma experiments it is important to have methods to measure neutrons [7]. In fusion with deuterium reactions, ${ }^{235} \mathrm{U}$ cameras are used to monitor the neutron flux, spectrometers with Stilbene scintillators to determine the neutron spectrum and to calibrate these detectors, ${ }^{252} \mathrm{Cf}$ source is used [8]. In certain 
experiments with fusion, the neutron fluence is usually of the order of $10^{14} \mathrm{n} / \mathrm{cm}^{2}$ and it is necessary to look for detectors that support this intensity of radiation [9].

Despite being a mature discipline, neutron detection is always important issue in several areas. Thus, updated measurements of neutrons produced in $\mathrm{U}^{235}(\mathrm{n}, \mathrm{f})$ reactions induced with neutrons from 0.7 to $20 \mathrm{MeV}$ have been reported [10]. Also, ${ }^{4} \mathrm{He}$ gas scintillators have been used to detect neutrons produced during the nuclear fission of natural uranium samples bombarded with $2.45 \mathrm{MeV}$ neutrons produced in deuterium-deuterium reactions [11].

In problems related with nuclear safety such as: the control and prevention of illicit traffic of Special Nuclear Materials, the characterization of transuranic waste, in safeguards, and in the decontamination and dismantling of nuclear facilities, it is important to measure neutrons. Ports, customs and access points in several countries use gantry detectors with ${ }^{3} \mathrm{He}$ proportional detectors; however, its scarcity worldwide has led to the need to search for new detection options [12, 13]. Guzmán-García et al, characterized the performance of scintillators of $\mathrm{ZnS}(\mathrm{Ag})$ and ${ }^{10} \mathrm{~B}$ and determined their response to neutrons produced by sources of ${ }^{241} \mathrm{AmBe}$ and ${ }^{252} \mathrm{Cf}$ [14]. Agreements on the control of nuclear weapons require inspection procedures where neutron detectors like those based on bubble detectors can be used [15].

Personal neutron dosimetry faces several challenges since there is a strong dependence between the angular and energy distribution of the neutrons with the coefficients of neutron fluence-to-dose conversion. The need to overcome these difficulties motivates the search for new solutions, thus in nuclear power plants have been used a naked ${ }^{3} \mathrm{He}$ thermal neutron detector and two spherical moderators (3 and 9 inches-diameter) to measure thermal, epithermal, and fast neutrons [16]. Around linear accelerators for medical uses silicon diodes type P-I-N have been used to verify the dose due to photoneutrons [17].

Regardless the area where neutrons are used is important to have a reliable method for measuring them and to estimate the dose. The need to measure neutrons has led to innovation in the detection of neutrons, thus Amaro et al, used ${ }^{10} \mathrm{BF}_{3}$ detectors where ${ }^{10} \mathrm{~B}$ was added in aerosol of nanoparticles [18]. Moderate ${ }^{3} \mathrm{He}$ detectors with high and low density polyethylene are used to measure neutrons produced in the delayed $\beta$ neutron decay or $\beta$-n emission, in order to have evidence that supports certain theories of astrophysics related to the abundance of the isotopes [19]. Large size liquid scintillators are used in experiments to measure solar neutrinos, proton decay, and dark matter [20]. The DarkSide-50 system has 30 tons of liquid scintillator where background signals, terrestrial and cosmic origin, are suppressed [21].
In the last decade, neutron detectors have been developed to be used in different conditions [22]. The use of liquid scintillators, when they are built in large sizes, have the disadvantage of liquid leaks; there are chemical risks in their handling, and their toxicity, among others; most of these difficulties are eliminated if plastic scintillators are used such as EJ-299-33A [23].

Commercially there are several types of scintillation liquids, such as the UltimaGold ${ }^{\mathrm{TM}} \mathrm{AB}$ scintillator liquid and the Optiphase Trisafe scintillator liquid. These are well known in the area of research for the detection of betas and alphas, they are used more routinely, to make measurements of environmental samples. The liquid scintillator UltimaGold ${ }^{\mathrm{TM}} \mathrm{AB}$ was used to optimize the determination of ${ }^{3} \mathrm{H}$ in aqueous samples [24]. Also, this scintillator was used to determine the concentration of Po-210, which is radiotoxic, in tobacco from India [25]. Broda et al, studied the influence of cocktail composition on the standardization of radionuclides [26]. Also, several scintillators were used to determine their response to ${ }^{3} \mathrm{H} /{ }^{14} \mathrm{C}$ [27]. Another type of commercial liquid scintillator has been used to measure the radon-to-radium ration [28], and in the area of biomaterials [29].

UltimaGold ${ }^{\mathrm{TM}} \mathrm{AB}$ and OptiphaseTrisafe scintillator liquids are marketed and promoted for the detection of alpha and beta particles. To be used, the sample must be mixed with the scintillator liquid, therefore both scintillators have low density. If $\gamma$-rays interact with these liquid scintillators, electrons will be produced due to photoelectric effect, Compton scattering and pair production, due to the low $\mathrm{Z}$ of scintillators the most probable interaction will be Compton scattering, where the scattered electron will induce scintillations. If fast neutrons collide with the scintillators $(n, p)$ reactions will be produced in the hydrogen and the proton will induce scintillations that will have different features than the scintillations induced by electrons. This difference will be noticed in the pulse height spectra, thus both scintillators can be used to detect $\gamma$-rays and neutrons. Therefore, the objective of this work was to determine the response to gamma rays and neutrons of two organic liquid scintillators that are commercialized as detectors for beta and alpha particles.

\section{Materials and Methods}

In this study, we used two scintillation liquids commercially promoted as alphas and betas detectors. These detectors are UltimaGold $^{\mathrm{TM}} \mathrm{AB}$ and OptiphaseTriSafe, the first is from PerkinElmer and the second is from the company EG \& G company. 
Both scintillation liquids are organic compounds whose content of carbon and hydrogen is high, therefore should detect gamma rays by the excitation produced by the electrons released in the scintillator and detect neutrons by the excitation produced by the protons released by fast neutrons in $(n, p)$ reactions with hydrogen in the same way that alphas induce scintillation.

In order to determine the response of both scintillators to gamma and neutron, two $25 \mathrm{ml}$ containers were prepared, one with each scintillator liquid. The containers are made of glass with low potassium content, since potassium absorbs the type of light that is emitted by the scintillator liquid. The liquid scintillator container was optically coupled to a photomultiplier tube (PMT) Dupont brand model 6292, as is shown in Figure 1 (a).

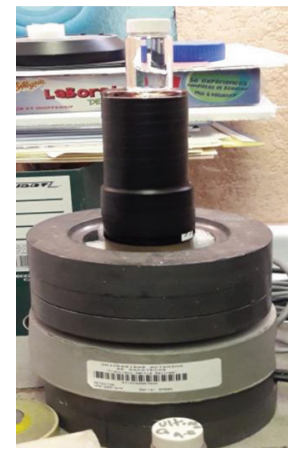

(a)

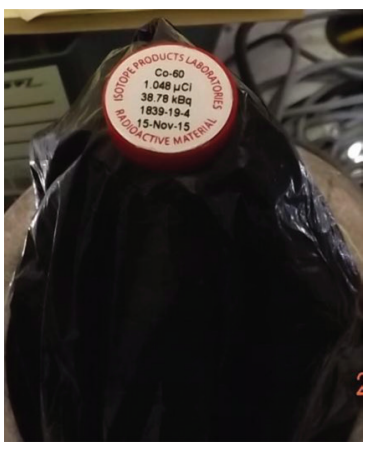

(b)
Figure 1: (a) Vial with the scintillator coupled to the PMT. (b) Measuring the response to ${ }^{60} \mathrm{Co}$ gamma-rays.

The PMT has a base made by EG \& G Ortec model 296. This array was connected into a spectrometric system with a high voltage supply, an amplifier, and a multichannel analyzer. The scintillator container and the PMT were isolated from the light using a thick black polyethylene bag and the response to gamma rays was carried out allocating the $\gamma$-ray source above the scintillator container, as is shown in Figure 1 (b).

The pulse height spectra of $0.511,0.662,0.834$, $1.17,1.27$, and $1.33 \mathrm{MeV} \gamma$-rays produced by ${ }^{137} \mathrm{Cs},{ }^{54} \mathrm{Mn}$, ${ }^{22} \mathrm{Na}$, and ${ }^{60} \mathrm{Co}$ sources was measured by both scintillators using the same measuring conditions. Gamma-ray sources were produced by Eckert \& Ziegler Isotope Products. On November $15^{\text {th }}, 2015$ the initial activity of the sources are shown in Table 1. For each of these sources, and for radiation background, the pulse height spectrum was measured in the multichannel analyzer.

In order to determine the response to neutrons, a source of ${ }^{241} \mathrm{AmBe}$ of $3.7 \times 10^{9} \pm 10 \% \mathrm{~Bq}$ was used. The pulse height spectrum due to neutrons was measured to 25 $\mathrm{cm}$ and $50 \mathrm{~cm}$ source-to-scintillator distances.
Background, gamma ray, and neutron pulse height spectra were measured using 1024 channels and during a live time of 1800 seconds, using the same electronics features conditions.

The first group of measurements was carried out with no reflector in the scintillator liquid containers. Then, glass containers were painted using nail polish in white, and background, $\gamma$-rays and neutrons pulse height spectra were measured again.

Table 1: Gamma ray sources initial activity

\begin{tabular}{lc}
\hline Sources & Initial activity $[\mathbf{k B q}]$ \\
\hline${ }^{137} \mathrm{Cs}$ & $38.92 \pm 3 \%$ \\
${ }^{54} \mathrm{Mn}$ & $40.03 \pm 3 \%$ \\
${ }^{22} \mathrm{Na}$ & $35.67 \pm 3 \%$ \\
${ }^{60} \mathrm{Co}$ & $38.79 \pm 3 \%$ \\
\hline
\end{tabular}

\section{Results}

In Figure 2 are shown the responses of UltimaGold ${ }^{\mathrm{TM}} \mathrm{AB}$ scintillator without reflector. The response is shown in terms of the pulse height spectra due radiation background, ${ }^{137} \mathrm{Cs}$, ${ }^{54} \mathrm{Mn},{ }^{22} \mathrm{Na}$, and ${ }^{60} \mathrm{Co} \gamma$-rays, and to neutrons produced by the ${ }^{241} \mathrm{AmBe}$ isotopic neutron source. The response to neutrons is to 25 and $50 \mathrm{~cm}$ from the scintillator.

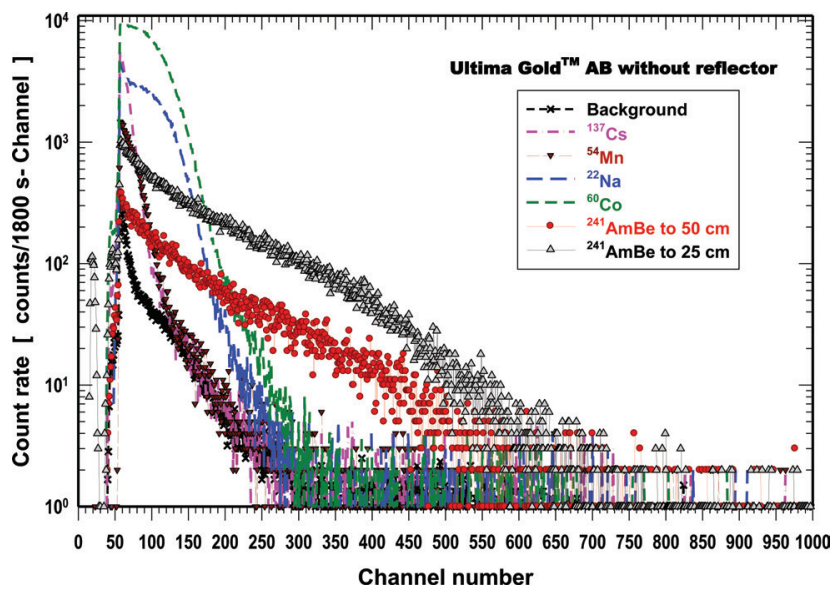

Figure 2: Pulse height spectra, due to background, $\gamma$-rays, and neutrons, measured with Ultima Gold $A B$ scintillator without reflector.

In Figure 3 are the pulse height spectra produced by the scintillator OptiphaseTrisafe without reflector, while in 
Figure 4 the responses of UltimaGold ${ }^{\mathrm{TM}} \mathrm{AB}$ scintillator with reflector. Responses are the pulse height spectra due to background, $\gamma$-rays, and neutrons.

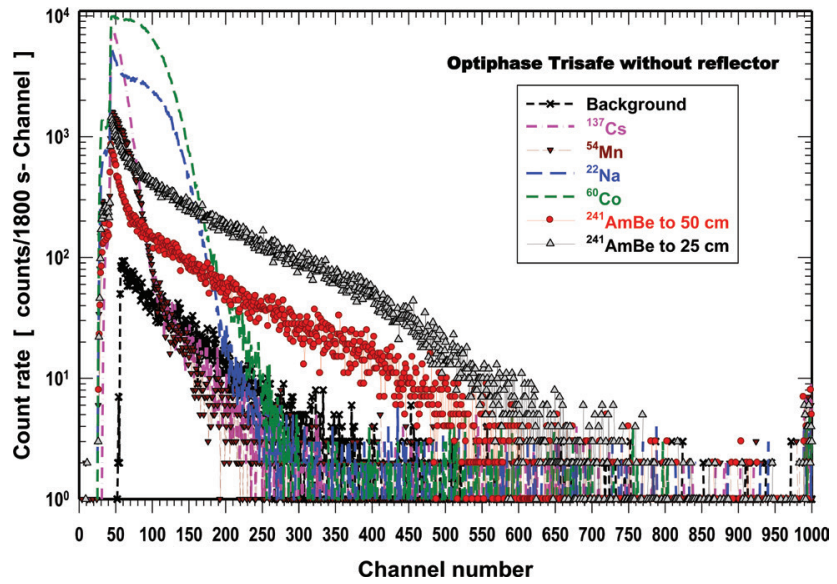

Figure 3: Pulse height spectra, due to background, $\gamma$-rays, and neutrons, measured with OptiphaseTrisafescintillator without reflector.

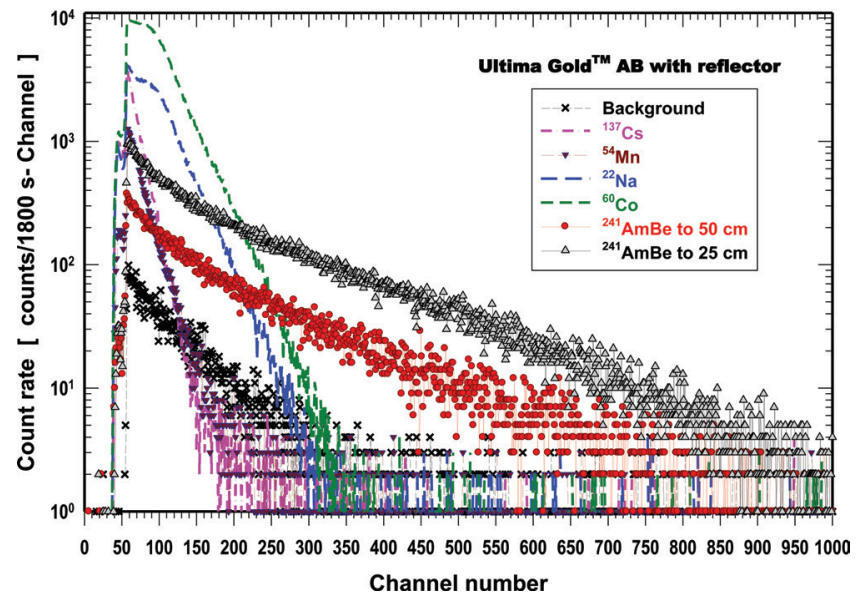

Figure 4: Pulse height spectra, due to background, $\gamma$-rays, and neutrons, measured with Ultima Gold $A B$ scintillator with reflector.

In Figure 5 are the pulse height spectra produced by the scintillator OptiphaseTrisafe with reflector.

In figure 6 are shown the pulse height spectra (PHS) of ${ }^{137} \mathrm{Cs}$ and ${ }^{60} \mathrm{Co} \gamma$-rays on UltimaGold ${ }^{\mathrm{TM}} \mathrm{AB}$ scintillator without and with reflector. For the OptiphaseTrisafe scintillator, without and with reflector, in figure 7 are shown the spectra of ${ }^{137} \mathrm{Cs}$ and ${ }^{60} \mathrm{Co} \gamma$-rays. In Figures 6 and 7 the PHS have been corrected by background.

The effect, on the PHS due to neutrons, of adding the reflector in both scintillators is shown in Figure 8 for UltimaGoldTM AB and in Figure 9 for OptiphaseTrisafe.

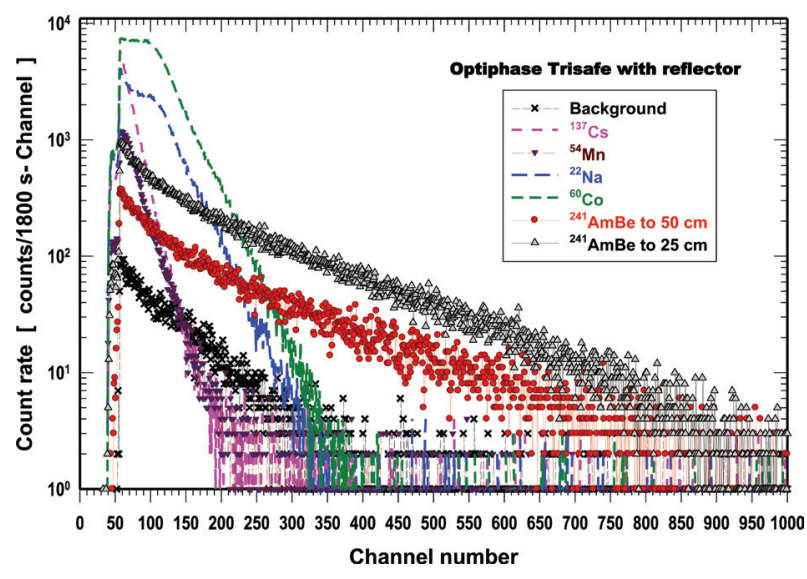

Figure 5: Pulse height spectra, due to background, $\gamma$-rays, and neutrons, measured with OptiphaseTrisafescintillator with reflector.

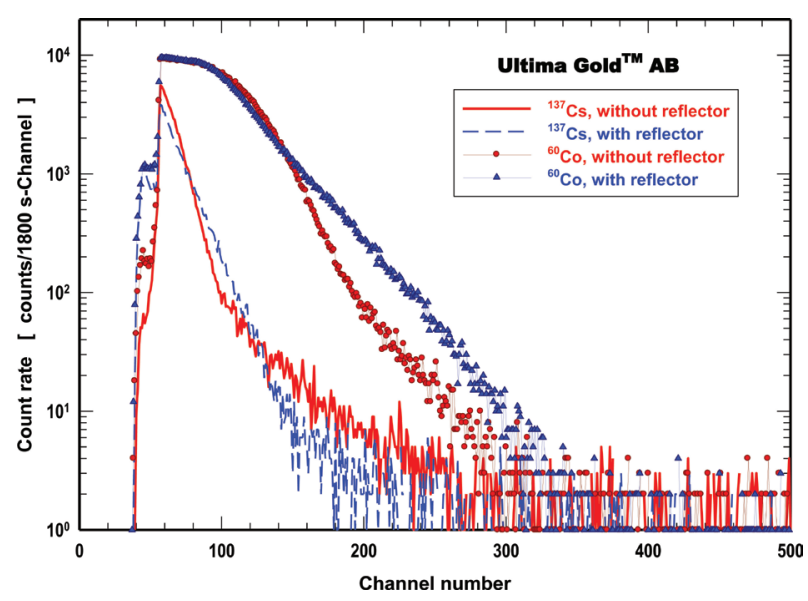

Figure 6: Background corrected pulse height spectra, due to ${ }^{137} \mathrm{Cs}$ and ${ }^{60} \mathrm{Co} \gamma$-rays, measured with UltimaGold ${ }^{\mathrm{TM}} \mathrm{AB}$, without and with reflector.

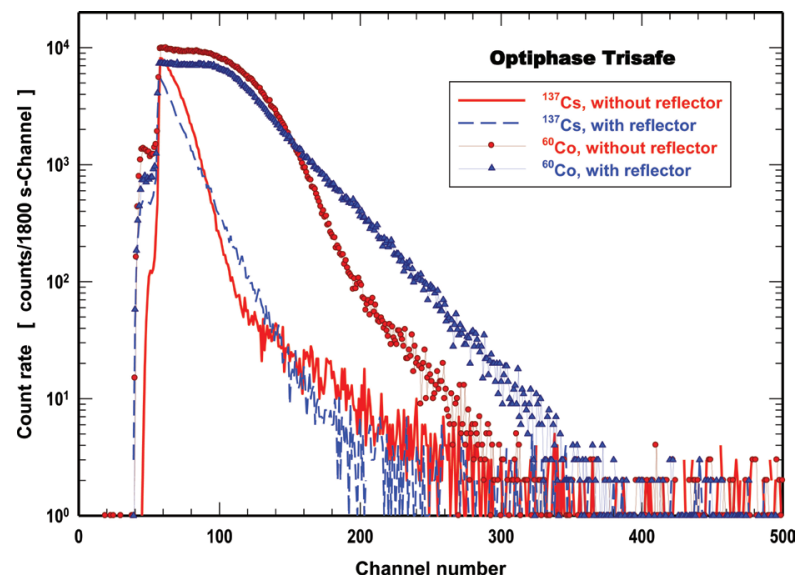

Figure 7: Background corrected pulse height spectra, due to ${ }^{137} \mathrm{Cs}$ and ${ }^{60} \mathrm{Co} \gamma$-rays, measured with OptiphaseTrisafe, without and with reflector. 


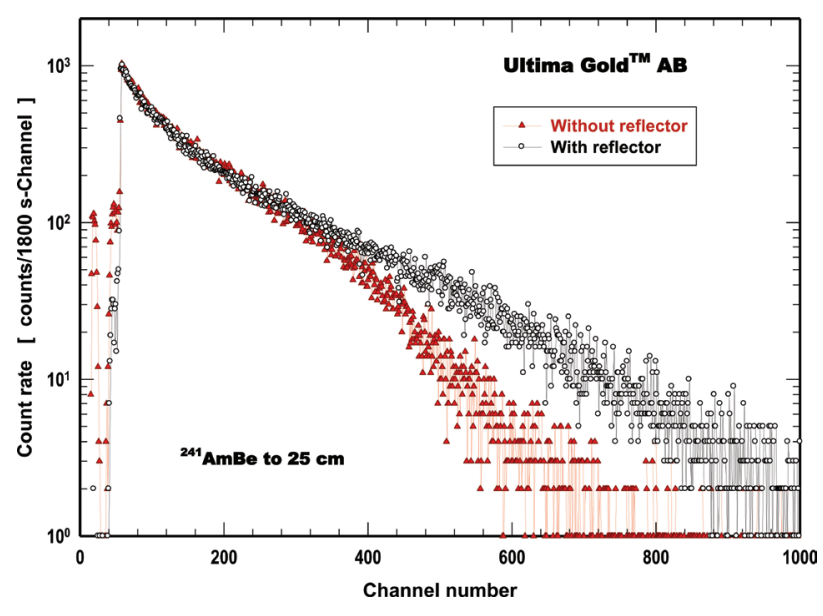

Figure 8: Background corrected PHS due to ${ }^{241} \mathrm{AmBe}$ neutrons measured with UltimaGold ${ }^{\mathrm{TM}} \mathrm{AB}$ scintillator without and with reflector.

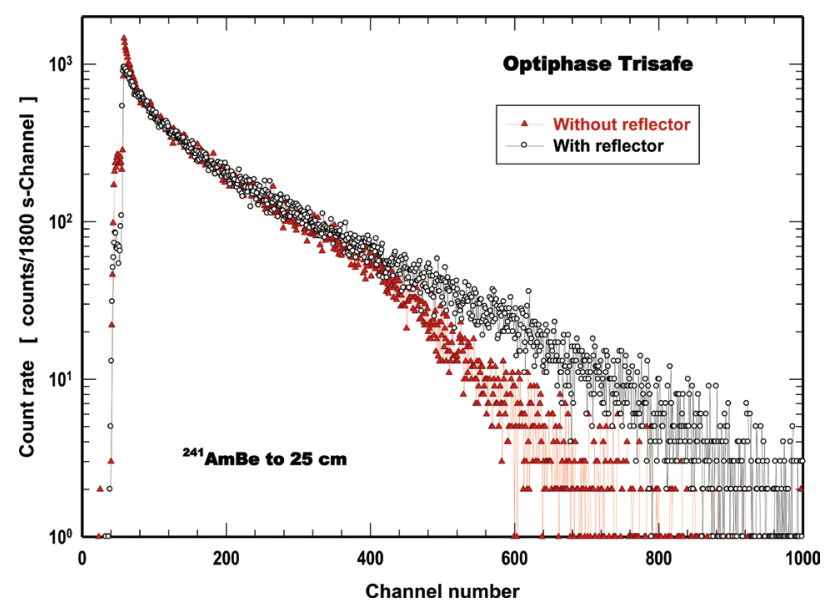

Figure 9: Background corrected PHS due to ${ }^{241} \mathrm{AmBe}$ neutrons measured with OptiphaseTrisafe scintillator without and with reflector.

\section{Discussion}

In the Figures 2 and 3 show the pulse height spectra for both scintillators, without reflector, for the background, gamma rays and neutrons. The pulse height spectra for neutrons are different to the pulse height spectra for $\gamma$-rays. In the responses to gamma-rays the Compton edge shifts to the right as the gamma ray energy increases. For $\gamma$-rays of ${ }^{60} \mathrm{Co}$ practically the pulse height spectrum ends in channel 350 .

In the case of neutron responses, for both scintillators the pulse height spectra have the same shape from channel 50 to channel 750 . However, the pulse height spectrum is greater when the source is to $25 \mathrm{~cm}$ than when it is to 50 $\mathrm{cm}$. This is due to the neutron flux at $25 \mathrm{~cm}$ is greater than the neutron flux at $50 \mathrm{~cm}$ distance. The response of the scintillators for gammas is different than the response for neutrons. This is because the interaction of the gamma rays is mainly with the electrons of the scintillators, while the interaction with neutrons occurs with the hydrogen of both scintillators.

In Figures 4 and 5 the pulse height spectra for background, $\gamma$-rays and neutrons for both scintillators with reflector are shown. Here can be noticed that the shape and end point of pulse height spectra for gamma-rays are the same as the case of scintillators without reflectors. The shape of pulse height spectra for neutrons looks alike the spectra measured without reflector, however, when the reflector is used the end point is in channel 1000 . Therefore, the use of reflector increases the amount of scintillations reaching the PMT.

The shape of pulse height spectra (PHS) for $\gamma$-rays and neutrons (Figures 2, 3, 4, and 5) are consistent with those reported by Wang, Seidaliev \& Mandapaka for their design of a neutron rem meter [30].

The PHS for ${ }^{241} \mathrm{AmBe}$ neutrons of both scintillators are similar to the pulse height spectra reported by Becchetti et al, although they used C6D6 scintillators; NE230, BC537 and EJ315 which are plastic scintillators, and an organic scintillator in liquid state of deuterated Xylene (C8D10; EJ301D) [31].

The responses of both scintillators to neutrons are similar to those reported for a plastic scintillator EJ-299$33 \mathrm{~A}$ that together with BC501A were irradiated with almost monoenergetic neutrons [23].

For both scintillators, without or with reflector, as the $\gamma$-ray energy increases the pulse height spectrum shifts to higher channels. For $0.662 \mathrm{MeV} \gamma$-rays can be noticed that approximately from channel 80 to channel 120 the pulse height spectrum is larger when is measured with the UltimaGold $^{\mathrm{TM}}$ with reflector. The same effect is shown for ${ }^{60} \mathrm{Co} \gamma$-rays where the increase is noticed approximately from channel 150 to channel 320 (Fig. 6). For Optiphase (Fig. 7) the same effect is noticed with the difference that for ${ }^{137} \mathrm{Cs}$ photons a larger amount of counts are notice from channel 90 to 140 approximately and from 160 to 340 for ${ }^{60} \mathrm{Co} \gamma$-rays.

The effect of adding the reflector is also noticed, for both scintillators, in the PHS corrected by background for the ${ }^{241} \mathrm{AmBe}$ neutrons. For both scintillators the spectrum measured with the reflector has a larger amount of counts per channel above channel 400. In the case of UltimaGold ${ }^{\mathrm{TM}}$ beyond channel 400 the count rates measured with the scintillator with reflector are larger than those obtained without the reflector (Fig.8). This difference is larger than the Optiphase Trisafe scintillator (Fig. 9). 


\section{Conclusions}

In this work have been measured the gamma and neutron responses of the two organic scintillation liquids, UltimaGold $^{\mathrm{TM}} \mathrm{AB}$ and OptiphaseTrisafe, which are promoted commercially to measure $\beta$ and $\alpha$ particles.

The two organic liquid scintillators, UltimaGold ${ }^{\mathrm{TM}} \mathrm{AB}$ and Optiphase Trisafe, in addition to be used to measure $\alpha$ and $\beta$ particles, can be used to measure $\gamma$-rays and neutrons, because the PHS due to $\gamma$-rays is different to the PHS due to neutrons.

The PHS due to gamma rays show the Compton edge that shifts to the right as the energy of the incident photon increases. As the photon energy increases the PHS endpoint shifts to the right. The end-point for ${ }^{60} \mathrm{Co} \gamma$-rays is around channel 350 .

The PHS due to neutrons has different shape to the PHS due to $\gamma$-rays. For neutrons the PHS end-point is in channel 1000.

For both scintillators the use of a white color reflector improves the amount of count rates in the PHS.

A limitation of this work was that to measure neutrons a ${ }^{241} \mathrm{AmBe}$ neutron source was used. Beside neutrons this source produce $4.4 \gamma$-rays due to ${ }^{12} \mathrm{C}^{*}$ decay that is produced during the $\operatorname{Be}(\alpha, n){ }^{12} \mathrm{C}^{*}$ reaction, and $59.5 \mathrm{keV} \gamma$-rays during the ${ }^{241} \mathrm{Am}$ decay. Also the neutron source is enclosed in a shell of polyethylene where neutrons are moderated and captured by $\mathrm{H}$ producing $2.2 \mathrm{MeV}$ photons. Therefore, in the neutron pulse height spectra count rates are due to gammas and neutrons. If the pulse shape discrimination technique is not available, the scintillators can be used to measure neutrons by assuming that beyond channel 400 the count rate are only due to neutrons.

\section{Acknowledgments}

M.I. Escalona-Llaguno, S.M. Sarmiento-Rosales, C.A. Márquez-Mata, and G.E. Campillo-Rivera thanks to the National Council of Science and Technology in Mexico (CONACyT) for the granted scholarship to pursue their postgraduate degree. Also, we thanks to the Council of Science, Technology and Innovation of Zacatecas $(\mathrm{COZCyT})$ for the constant support in our participation in national and international meetings.

\section{References}

[1] V. A. Baskov and V. V. Polyansky, Bull. Lebedev Phys. Inst. 45, 123 (2018).

[2] H. R. Vega-Carrillo, V. M. Hernández-Dávila, T. Rivera and A. Sánchez, Rad. Phys. Chem. 95, 122 (2014). https://doi.org/10.1016/j.radphyschem.2013.05.001.
[3] J. C. McDonald, B. R. L. Siebert and W. G. Alberts, Nucl. Instrum. Meth. Phys. Res. A 476, 347 (2002). https://doi.org/10.1016/S0168-9002(01)01460-7.

[4] G. F. Knoll, Radiation Detection and Measurement. (John Wiley \& Sons, Michigan, 2010).

[5] A. J. Peurrung, Nucl. Instrum. Meth. Phys. Res. A 443, 400 (2000). https://doi.org/10.1016/S0168-9002(99)01165-1.

[6] A. V. Zhirkin et al., Nucl. Fus. 57, 066044 (2017). https://doi.org/10.1088/1741-4326/aa69d4.

[7] T. Nishitani et al., Fus. Eng. Des. 136, 210 (2018). https://doi.org/10.1016/j.fusengdes.2018.01.053.

[8] T. Nishitani, K. Ogawa and M. Isobe, Fus. Eng. Des. 123, 1020 (2017). https://doi.org/10.1016/j.fusengdes.2017.02.038.

[9] L. Liu, A. Liu, S. Bai, L. Lv, P. Jin and X. Ouyang, Sci. Rep. 7, 13376 (2017). https://doi.org/10.1038/s41598-017-13715-3.

[10] M. J. Devlin et al., Nucl. Data Sheets 148, 322 (2018). https://doi.org/10.1016/j.nds.2018.02.008.

[11] T. Zhu et al., Nucl. Instrum. Meth. Phys. Res. A 848, 137 (2017). https://doi.org/10.1016/j.nima.2016.12.016.

[12] R. V. D. Ryzhikov, S.V. Naydenov, T. Pochet, G. M. Onyshchenko, L.A. Piven and C.F. Smith, IEEE Trans. Nucl. Sci. 65, 2547 (2018). https://doi.org/10.1109/TNS.2018.2825642.

[13] K. A. Guzmán-García, H. R. Vega-Carrillo, E. Gallego, J. A. González, R. Méndez,-Villafañe, A. Lorente and S. Ibañez-Fernandez, Rad. Meas. 107, 58 (2017). https://doi.org/10.1016/j.radmeas.2017.11.001.

[14] K. A. Guzmán-García, H. R. Vega-Carrillo, E. Gallego, A. Lorente, R. Méndez-Villafañe J. A. González and S. Ibañez-Fernandez, Appl. Rad. Isot. 117, 58 (2016). https://doi.org/10.1016/j. apradiso.2016.03.015.

[15] F. D'Errico, A. Chierici, M. Gattas-Sethi, R. Goldston and A. Glaser, Rad. Prot. Dosim. 180, 210 (2018). https://doi.org/10.1093/rpd/ncy037.

[16] O. V. Hoey, F. Vanhavere and L. Verbraeken, Rad. Prot. Dosim. 180, 85 (2018). https://doi.org/10.1093/rpd/ncx224.

[17] V. Gracanin et al., Rad. Meas. 106, 391 (2017). https://doi.org/10.1016/j.radmeas.2017.01.004.

[18] F. D. Amaro, C. M. B. Monteiro, J. M. F. dos Santos and A. Antognini, Sci. Rep. 7, 41699 (2017). https://doi.org/10.1038/srep41699.

[19] J. L. Tain et al., Acta Phys. Pol. B 49, 417 (2018). https://doi.org/10.5506/APhysPolB.49.417. 
[20] Miramonti, L., International Journal of Modern Physics A 32, 1743010 (10 pages) (2017). https://doi.org/10.1142/S0217751X17430102.

[21] P. Agnes et al., J. Instrum. 12, T12004 (2017). https://doi.org/10.1088/1748-0221/12/12/T12004.

[22] I. Mor, D. Vartsky, V. Dangendorf, K. Tittelmeier, M. B. Goldberg, D. Bar and M. Brandis, J. Instrum. 12, C12022 (2017). https://doi.org/10.1016/j.nima.2018.02.113.

[23] P. Roy, K. Banerjee, A. K. Saha, C. Bhattacharya, J. K. Meena, P. Bhaskar, S. Mukhopadhyay and S. Bhattacharya, Nucl. Instrum. Meth. Phys. Res. A 901, 198 (2018). https://doi.org/10.1016/j.nima.2018.06.007.

[24] P. L. Pujol and J. A. Sanchez-Cabeza, J. Radioanal. Nucl. Chem. 242, 391 (1999). https://doi.org/10.1007\%2FBF02345568.

[25] A. Srivastava, V. Tuli and U. W. Scherer, Radiochim. Acta 106, 787 (2018). https://doi.org/10.1515/ract-2017-2848.
[26] R. Broda, K. Małetka, T. Terlikowska and P. Cassette, Appl. Rad. Isot. 56, 285 (2002). https://doi.org/10.1016/S0969-8043(01)00202-0.

[27] F. Verrezen, H. Loots and C. Hurtgen, Appl. Rad. Isot. 66, 1038 (2008). https://doi.org/10.1016/j.apradiso.2008.02.050.

[28] S. P. D. Bhade, P. J. Reddy, S. Anilkumar, R. K. Singhal and D. D. J. Rao,. Radioanal. Nucl. Chem. 315, 13 (2018). https://doi.org/10.1007/s10967-017-5643-x.

[29] H. Kafa, J. T. W. Wang, N. Rubio, K. Venner, G. Anderson, E. Pach, B. Ballesteros, J. E. Preston, N. J. Abbott and K. T. Al-Jamal, Biomaterials 53, 437 (2015). https://doi.org/10.1016/j.biomaterials.2015.02.083.

[30] C. C. Wang, M. Seidaliev and A. Mandapaka, Health Phys. 94, 440 (2008). https://doi.org/10.1097/01. HP.0000300493.53385.5d.

[31] F. D. Becchetti, R. S. Raymond, R. O. Torres-Isea, A. Di Fulvio, S. D. Clarke, S. A. Pozzi and M. Febbraro, Nucl. Instrum. Meth. Phys. Res. A 820, 112 (2016). https://doi.org/10.1016/j.nima.2016.02.058. 


\section{Journal of Nuclear Physics, Material Sciences, Radiation and Applications}

Chitkara University, Saraswati Kendra, SCO 160-161, Sector 9-C, Chandigarh, 160009, India

Volume 6, Issue 2

February 2019

ISSN 2321-8649

Copyright: [C 2019 Hector Rene Vega-Carrillo et al.] This is an Open Access article published in Journal of Nuclear Physics, Material Sciences, Radiation and Applications (J. Nucl. Phy. Mat. Sci. Rad. A.) by Chitkara University Publications. It is published with a Creative Commons Attribution- CC-BY 4.0 International License. This license permits unrestricted use, distribution, and reproduction in any medium, provided the original author and source are credited. 